\title{
Artist-Directable Real-Time Rain Rendering in City Environments
}

\author{
Natalya Tatarchuk* and John Isidoro** \\ ATI Research
}

\section{Introduction}

Many of the recent games incorporating rain rendering in their worlds use a very simplistic approach: rendering stretched, alphablended particles to simulate falling rain drops. This approach fails to create a truly convincing and interesting rain impression. Typically, the games only include one or two effects such as the stretched rain particles and perhaps a simple CPU-based water puddle animation to simulate the impression of rainy environment. This results in an unrealistic environment with the rain not reacting correctly to light effects, such as lightning or spotlights.

We present a number of novel techniques for rendering both particle-based rain and rain based on a post-processing imagespace technique, as well as many additional secondary rain effects, without which one cannot generate a true immersive rain environment. Our techniques provide a variety of artist-directable controls and respect the rules of physics for simulating rainfall. They utilize light reflection models to allow the rain to respond dynamically and correctly to the lighting changes in the complex environment of the ATI "ToyShop" $"[1]$ demo due to illumination from atmospheric effects (for example, lightning).

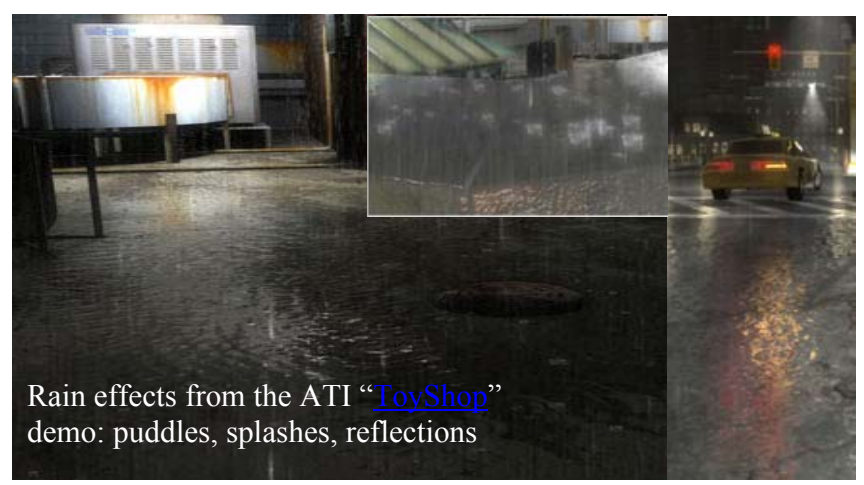

\section{Realistic Rain Rendering}

We developed a novel post-processing rain effect simulating multiple layers of falling raindrops in a single compositing pass over the rendered scene. We create motion parallax for raindrops utilizing projective texture reads. The illumination for rain is computed using water-air refraction for individual raindrops as well as reflection due to surrounding light sources and the Fresnel effect. We provide a set of artist knobs for controlling rain direction and velocity, and the rainfall strength. The raindrop rendering receives dynamically-updated parameters such as lightning brightness and direction from the lightning system to allow correct illumination resulting from lightning strikes.

To simulate raindrops falling off various objects in our scene, we use billboard particle systems with normal-mapped rain droplets. The illumination model used for these particles is the same as for the composite rain. To control raindrop transparency, we attenuate raindrop opacity by their distance in the scene. Additionally, we increase raindrop transparency when the lightning strikes to maintain physical illusion of water. In our scenes we found that using on the order of 10-15,000 particles in a complex scene gives excellent results.

\footnotetext{
*natasha@ati.com

**jisidoro@ati.com
}

We simulate raindrops splashing when hitting solid objects by colliding individual particles with objects in the scene. A single filmed high-quality splash sequence for milk drop was used to drive the raindrop splash event. We integrated a high degree of randomization for particle parameters, and dynamically flipped horizontal texture sampling for the filmed sequence. For illuminating the raindrop splashes, we added backlighting to the splashes so that they appear to correct respond to the environment lights (and thus display the subtle effects of raindrops splashing under a street light).

\section{GPU-Based Water and Droplet Movement}

The rain drop particle collisions generate ripples in rain puddles in our scene. We use an implicit integration scheme to simulate fluid dynamics for rendering dynamically lit puddle ripples. Similar to real-life raindrops, in our system we generate multiple ripples from a single raindrop source which interact with other ripples on the water surface. The physics simulation for water movement is done entirely on the GPU. We treat the water surface as a thin elastic membrane, computing forces due to surface tension and displacing water sections based on the pressure exerted from the neighboring sections. Our system provides simple controls to the artists to specify water puddle placement and depth.

We adopted the offline raindrop simulation system from Kaneda et al ${ }^{[2]}$ to the GPU to convincingly simulate and render water droplets trickling down on glass planes in real-time. This system allows us to simulate the quasi-random meandering of raindrops due to surface tension and the wetting of the glass surfaces due to water trails left by droplets passing on the window. Our system produces a correctly lit appearance including refraction and reflection effects.

\section{View-Dependent Streaky Reflections}

Realistic streaky reflections increase the feel of rain on wet streets and various object surfaces. These reflections are very prominent in any rainy scene and appear to stretch toward the viewer. To simulate the appearance of a wet city street in the rainy night, we render image-space blurry reflections on wet objects.

To simulate view-dependent warped reflections, we render proxy geometry into a reflection buffer and dynamically stretch the reflected objects view-dependently in a vertex shader program. We utilize an expanded dynamic range for representing the rendered colors so that we can preserve the brightest lights for reflections (such as street lamps or taxi headlights). We use a post-processing technique to dynamically streak the reflection buffer in the vertical direction to simulate warping due to raindrops striking in the puddles. This aids in removing specular aliasing and excessive flickering from reflections which would otherwise be highly distracting otherwise.

All of these combined effects allow us to create a very convincing, realistic impression of rainy night in a cityscape at highly interactive rates.

\section{References}

[1] ATI "ToyShop" demo (video and executable) can be found at http://www.ati.com/developer/demos/rx1800.html

[2] KANEDA, K., IKEDA, S., YAMASHITA, H. 1999 "Animation of Water Droplets Moving Down a Surface", The Journal of Visualization and Computer Animation, pp. 15-26 